\title{
Effects of shape variations on the energy metabolism of the sand cricket Gryllus firmus: a geometric morphometric analysis
}

\author{
Roberto F. Nespolo ${ }^{1}{ }^{*}$, Roger D. Sepúlveda ${ }^{1}$, Luis E. Castañeda ${ }^{1}$ and Derek A. Roff ${ }^{2}$ \\ ${ }^{1}$ Instituto de Ecología y Evolución, Facultad de Ciencias, Universidad Austral de Chile, Casilla 567, Valdivia, Chile. \\ 2 Department of Biology, University of California, Riverside, CA 92521, USA.
}

\begin{abstract}
Respiration and energy metabolism are key processes in animals, which are severely constrained by the design of physical structures, such as respiratory structures. Insects have very particular respiratory systems, based on gas diffusion across tracheae. Since the efficiency of the tracheal respiratory system is highly dependent on body shape, the pattern of morphological variation during ontogeny could have important metabolic consequences. We studied this problem combining through-flow respirometry and geometric morphometrics in 88 nymphs of the sand cricket, Gryllus firmus. After measuring $\mathrm{CO}_{2}$ production in each individual, we took digital photographs and defined eight landmarks for geometric morphometric analysis. The analysis suggested that ontogenic deformations were mostly related to enlargement of the abdomen, compared to thorax and head. We found that (controlling for body size) metabolic variables and especially resting metabolism are positively correlated with a shape-component associated to an elongation of the abdomen. Our results are in agreement with the mechanics of tracheal ventilation in orthopterans, as gas circulation occurs by changes in abdominal pressures due to abdominal contractions and expansions along the longitudinal axis.
\end{abstract}

Key words: Shape variations, metabolic rate, morphology, insect respiration, orthopter, sand crickets.

\section{INTRODUCTION}

One of the most important physiological aspects in terrestrial animals is gas-exchange, for which adaptive evolution has provided a range of solutions, with their respective costs and benefits. For example, mammalian lungs are efficient respiratory organs as long as oxygen concentration is not limiting (Bramble and Jenkins, 1993). Avian lungs can cope with hypoxia, but at the cost of high desiccation risk (Willmer et al., 2005). Cutaneous respiration works well in air as long as the metabolic rate is low and/ or the environment is relative humidity-saturated (Feder and Burggren, 1985). Insects, on the other hand, have extremely efficient respiratory systems provided that body sizes are small (Suarez, 1998; Lehmann et al., 2000; Chown and Nicolson, 2004). The gas diffusion efficiency of the tracheal system of insects can be approximated very closely by the geometry of anastomising tubes (Snodgrass, 1993; Chown and Nicolson, 2004; Harrison et al., 2005). Hence, size and shape attributes are probably important limitations to the efficiency of insect respiration. In hemimetabolous species, during nymphal growth morphology and especially body proportions experience subtle changes between instars. According to Harrison et al. (2005), the efficiency of tracheal respiratory systems is highly dependent on the geometry of body parts during ontogeny. Several authors have studied the anatomic changes that occur in insect respiratory systems during ontogeny, and their functional consequences because of the enlargement of the tracheal system (Dixon and Kindlmann, 1999; Merrick and Smith, 2004; Harrison et al., 2005), but no study has addressed how shape variations (i.e., geometrical deformations independent of size) of the whole-insect body during nymphal growth are related to energy metabolism.
Four possibilities can be formulated regarding ontogeny, body shape and whole animal metabolism in insects. First, body proportions do not change (i.e., isometric growth), so that the differential contributions of different body parts to energy metabolism are constant during growth. Second, there is isometric growth, but there are allometric changes in metabolism due to changing differential contributions to energy metabolism by change in the distribution of tissues (e.g., ovarioles in females). Third, body proportions change (i.e., allometric growth, Gould, 1966), without consequences on metabolism (i.e., deformations occur without detectable consequences in whole-animal metabolism). Fourth, body proportions change during growth (i.e., deformations and/ or allometric growth occur) with measurable consequences on energy metabolism. In the latter case, a significant correlation between specific-shape coordinates and metabolism (independent of overall size) should be obtained, indicating which kind of deformation is correlated with energy metabolism, and what body part is responsible for it. Previous attempts to determine such associations have been performed using traditional morphometrics, with some success (Nespolo et al., 2005). However, the traditional approach does not capture subtle shape-variation, controlling for size effects. Geometric morphometrics, on the other hand, permits removing variation in translation, rotation and scale, and superimposes the objects in a common coordinated system. Consequently, in the current study we used geometric morphometrics to tackle the problem of shape variation and metabolism during ontogeny. We used flow-through respirometry to address the association between energy metabolism and geometrical deformations of nymphs (i.e., immature developmental stages) of a wide range of body sizes of the sand cricket Gryllus firmus.

\footnotetext{
${ }^{*}$ Corresponding author. Roberto F. Nespolo. Instituto de Ecología y Evolución, Facultad de Ciencias, Universidad Austral de Chile, Casilla 567, Valdivia, Chile. Phone: +56-63-221704, Fax: +56-63-221344, E-mail: robertonespolo@uach.cl
} 


\section{MATERIALS AND METHODS}

Insects

The inbred lines used in the present experiment were derived from a stock culture that originated from approximately 20 males and 20 females collected in northern Florida in 1981. The stock culture is maintained with a standing adult population of several hundred individuals (generally 100-500 adults, with occasional bottlenecks in which the population may have declined to about 50 adults). To prevent diapause, the temperature is maintained in excess of $25^{\circ} \mathrm{C}$. Nymphs and adults in both the stock and the experiment were fed Purina rabbit chow. Sand crickets exhibit one of the most variable life cycles known among field crickets. During most of the year, females lay eggs that hatch in a couple of weeks at room temperature. Nymphal development is variable as well, depending strongly on temperature and humidity. In this study, we used 88 nymphs ranging from 0.3 to $1.5 \mathrm{~g}$, which includes nearly all nymphal stages. These data were previously analyzed for a different purpose and published elsewhere (Nespolo et al., 2005).

Respirometry

Our respirometry system was similar to Lighton and Turner (2004) and Rogowitz and Chappell (2000). In brief, carbon dioxide production was measured continuously with an infra-red $\mathrm{CO}_{2}$ analyzer (LI6251, LI-COR Bioscience) capable of resolving differences of 0.2 parts per million ( $\mathrm{ppm}$ ) of $\mathrm{CO}_{2}$ in air. The analyzer was calibrated periodically against a precision gas mixture (i.e., span calibration) and against zero $\mathrm{CO}_{2}$ concentrations. There was almost no drift between calibrations. Flow rates of dry, $\mathrm{CO}_{2}$-free air were maintained at $\pm 1 \%$ by a mass-flowmeter (Sierra Instruments). Air was drawn from the ambient, water vapour and $\mathrm{CO}_{2}$ water scrubbed with a Drierite-Ascarite column, and flow rate controlled at $95 \mathrm{ml}$ $\mathrm{min}^{-1}$. The metabolic chamber was a $40 \mathrm{ml}$ glass-cylinder. To monitor movement we used a Sable System AD-1 activity detector, which detects the presence or absence of activity (i.e. it does not give a metric measurement of activity). Each cricket was measured for 45 minutes, but we considered only the last 20 minutes. Each record was automatically transformed by a macro program recorded in the Datacan software (Sable Systems International), in order to (1) to correct the six-second lag introduced by the distance between the analyzer and the chamber and then to match the activity record with the $\mathrm{CO}_{2}$ record, and (2) to transform the measurement from ppm of $\mathrm{CO}_{2}$ to $\mathrm{ml}-\mathrm{CO}_{2}$ per hour $\left(\mathrm{VCO}_{2}\right)$, taking into account the flow rate. To do this, we used the standard equation when the flowmeter was downstream of the animal chamber and CO2 was scrubbed prior to flow measurement:

$\mathrm{VCO} 2=\mathrm{STP}^{*}\left(\mathrm{FeCO}_{2}-\mathrm{FiCO}_{2}\right) * \mathrm{FR} /\left(1-\mathrm{FeCO}_{2}+\mathrm{FiCO}_{2} / \mathrm{RQ}\right)$, where

$\mathrm{STP}=$ standard temperature and pressure correction (equal to one with mass-flow controllers), $\mathrm{FeCO}_{2}=$ excurrent fractional concentration of $\mathrm{CO}_{2}, \mathrm{FiCO}_{2}=$ incurrent fractional concentration of $\mathrm{CO}_{2}, \mathrm{FR}=$ flow rate, $\mathrm{RQ}=$ respiratory quotient, which for herbivorous animals is assumed to be 0.85 . We took four respirometric variables from each individual: average, resting, maximum and minimum rates. As a "general" measure of metabolism, we took the complete average of each transformed record (AVG). In addition, we used the activity measurements to detect "resting" periods in each record. Most of the individuals presented a fairly clear pattern of resting and active periods (Fig. 1), revealed by the conspicuous drops in $\mathrm{VCO}_{2}$ during periods of undetectable activity (Fig. 1). Resting metabolism (REST) was computed as the average of the one-minute steady state $\mathrm{VCO}_{2}$ production during periods of inactivity. In addition, we considered the single maximum (MAX) and minimum (MIN) values of $\mathrm{VCO}_{2}$ during the record.

\section{Geometric morphometrics}

After each respirometric trial, crickets were fixed in alcohol (95\%) to assure maintaining their in vivo external morphology. The same day, digital images from a dorsal view were taken from each nymph in order to draw morphological landmarks on the digital photographs. Eight homologous landmarks were digitalized on each photograph using tpsDig2 (Rohlf, 2005a - Fig. 2). These landmarks were selected in order to capture the deformations that occur during ontogeny (i.e., disproportionate enlargement of the abdomen in relation to thorax and head) (see Harrison et al., 2005; Nespolo et al., 2005). These shape landmarks were transformed to an $\mathrm{x}-\mathrm{y}$ coordinate system following methods proposed by Rohlf and Slice (1990) and Adams (1999). Landmark coordinates were aligned using Generalized Procrustes Analysis (GPA). GPA is an important procedure because it removes variation in translation, rotation and scale, and superimposes the objects in a common (though arbitrary) coordinate system. In other words, size variations were controlled in order to compare shapes alone. Additionally, the aligned specimens from GPA provide points that can be projected into a Euclidean space that is tangent to the space of shapes (Bookstein, 1991; Adams et al., 2004; see details in Rohlf and Slice, 1990; Rohlf, 1999; Slice, 2001). In order to characterize graphically the morphological shape variation of the nymphs, small and large crickets were compared with a mean consensus configuration, which is used as a population mean to make

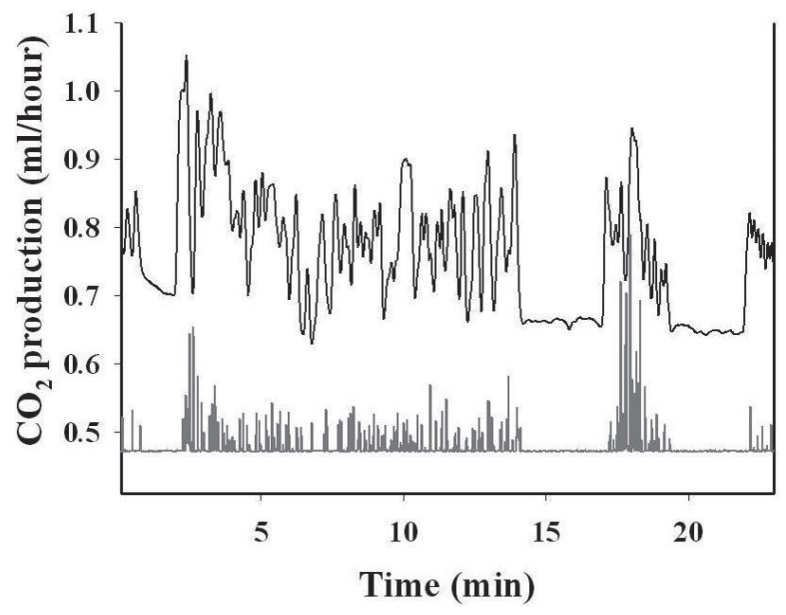

Figure 1. Representative $\mathrm{VCO}_{2}$ record (black line) showing maximun (a) and minimun (b) metabolism records combined with activity measurements (gray line) showing periods of resting (c) and activity (d) of the nymphs of the sand-crickets Grillus firmus. 
sure that the patterns of variation are not just artifacts of the superimposition (Rohlf and Slice, 1990). This comparison was performed through deformation grids to clearly visualize the distortion of the landmarks between small (young) and large (old) nymphs (Adams et al., 2004).

In order to obtain optimal shape variables (i.e., relative warps, which include both linear and non-linear variations), the aligned specimens were compared with the tpsRelw program (Rohlf, 2005b) using $a=1$, which accounted for most global (all landmarks are involved) and local (only some landmarks are involved) variations. Each relative warp is an orthogonal component (non-correlated among them) and therefore contains a unique and independent variance (i.e., analog to the axes in principal component analysis). The maximum number of relative warps is calculated from the equation $R w=2 \times k-4$, where $k=$ number of landmarks, times two ( $x-y$ coordinates) minus four degrees of freedom (two translations, $\mathrm{x}$ - and y-plane; one to match with other specimens rotation; and one scale to match with the size of other specimens). To establish the importance of each landmark on shape variations, we used eigenvectors (analog to factor loading), which correspond to vectors (with magnitude and direction) that account for the importance of each $x-y$ coordinate on each relative warp.

\section{Statistics}

The metabolic variables were average metabolism (AVG), maximum metabolic rate (MAX), minimum metabolic rate (MIN), resting metabolism (REST) and activity metabolism (ACT). They were standardized by body mass and then related to the relative warps by Pearson's correlation coefficients, in order to explore the relationships between the two sets of variables. Only the relative warps that showed significant

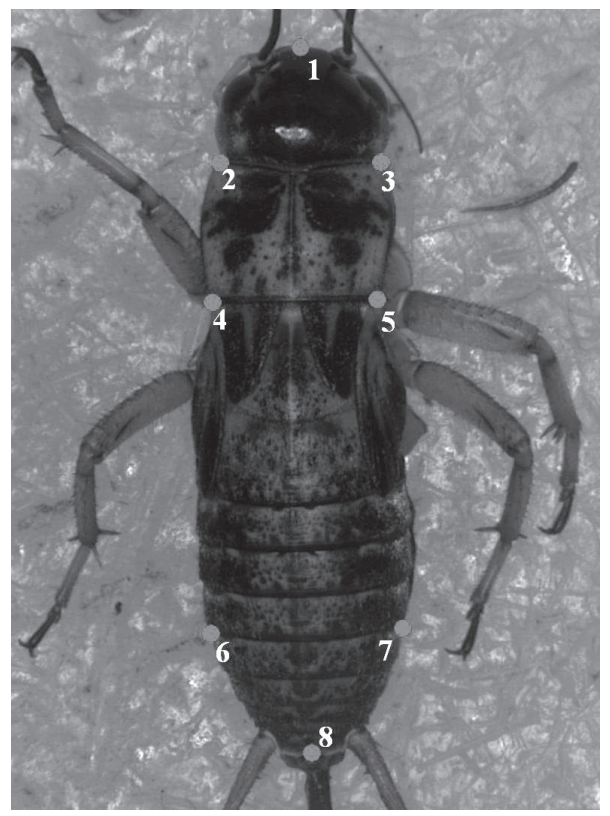

Figure 2. Morphological landmarks defined for the geometric morphometrics analysis of the nymphs of the sand-cricket Grillus firmus. correlations with at least one metabolic variable were used in a predictive model of regression analysis. Considering the absence of correlation among relative warps, we performed a multiple regression analysis (stepwise) using relative warps as predictor variables and each metabolic variable as dependent variable, in order to test whether metabolic variables were significantly explanatory of either attribute of body shape (Zar, 1999).

\section{RESULTS}

The five metabolic variables and body mass variation are presented in Table 1 . The mean consensus configuration from the $x-y$ coordinate overlaps and its displacement in the ontogenic gradient (ranging from small to large crickets) is shown in Fig. 3. The variations in small crickets display movements of landmarks on the thorax and abdomen, due

\section{TABLE 1}

Descriptive statistics (mean, standard deviation, standard error and individuals number) and of body mass (Log BM) and metabolic variables of the sand cricket Grillus firmus. For see materials and methods for abbreviations

\begin{tabular}{ccccc}
\hline & Mean & S.D. & S.E. & $\mathrm{n}$ \\
\hline Log BM & 0.260 & 0.163 & 0.017 & 89 \\
AVG & 0.095 & 0.060 & 0.006 & 89 \\
MIN & 0.014 & 0.011 & 0.001 & 89 \\
MAX & 0.149 & 0.090 & 0.010 & 89 \\
REST & 0.023 & 0.018 & 0.002 & 73 \\
ACT & 0.086 & 0.079 & 0.008 & 89 \\
\hline
\end{tabular}

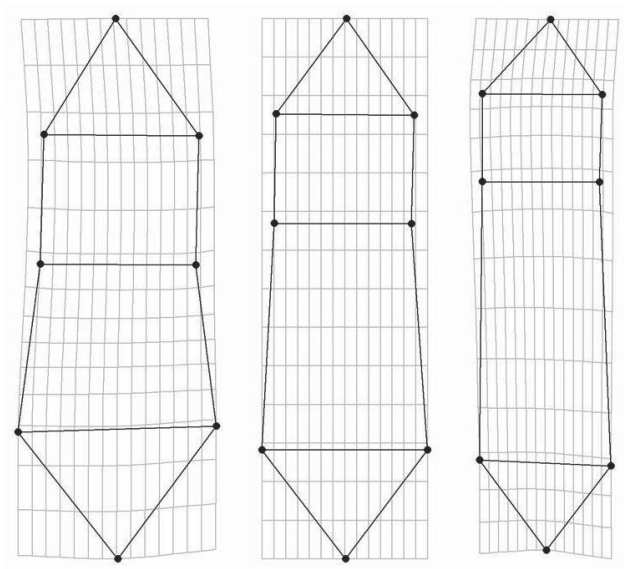

Figure 3. Deformation grids showing small (left draw) and large (right draw) crickets in reference with the mean consensus configuration (middle). In small crickets, the landmarks displacement shows short and wide thorax and abdomen, and large head. In large crickets, long abdomen makes the head and thorax appear disproportionately small (see also Fig. 2). The draws had been magnified $3 \mathrm{X}$ and some landmarks had been connected by lines to facilitate the interpretation of the differences, and therefore they do not represent real distances between landmarks. 
TABLE 2

Total relative contribution (SS = sum of squares), variance for the $x-y$ coordinate $\left(S^{2} x\right.$ and $\left.S^{2} y\right)$ and pooled variance $\left(S^{2} p\right)$ for eight landmarks (LM\#) defined in the geometric morphometrics analysis of the sand crickets nymphs

\begin{tabular}{ccccc}
\hline LM\# & SS & $\mathrm{S}^{2} x$ & $\mathrm{~S}^{2} y$ & $\mathrm{~S}^{2} p$ \\
\hline 1 & 0.0913 & 0.0001 & 0.0005 & 0.0006 \\
2 & 0.2075 & 0.0002 & 0.0001 & 0.0003 \\
3 & 0.2129 & 0.0002 & 0.0001 & 0.0003 \\
4 & 0.2080 & 0.0002 & 0.0006 & 0.0008 \\
5 & 0.2151 & 0.0004 & 0.0005 & 0.0009 \\
6 & 0.0229 & 0.0002 & 0.0002 & 0.0004 \\
7 & 0.0234 & 0.0002 & 0.0003 & 0.0005 \\
8 & 0.0190 & 0.0001 & 0.0006 & 0.0007 \\
\hline
\end{tabular}

to the variability produced by the landmarks $4-5$ and 6-7 respectively, generating a shortening of the abdomen (Fig. 3 left). In large crickets, the points associated with the landmarks 4-5 and 6-7 change direction, producing an opposite situation with the small crickets, a shortening of the thorax (Fig. 3 right). This shortening of the thorax is accompanied by a conspicuous elongation of the abdomen and a shortening of the caudal portion of crickets during growth (Fig. 3).

The geometric morphometric analysis on all shape variables ( $R w 1$ to $R w 12$ ) showed that the most important landmarks contributing to morphological variation in the crickets were those related to intersection points between the head and thorax (landmarks 2 and 3), and intersection points between the thorax and abdomen (landmarks 4 and 5) (Table 2). The relationship between shape and metabolic variables showed that only the relative warps 1, 4, 8, 9 and 10 were correlated with at least one metabolic variable, being the Rw4 the sole variable significantly correlated to all metabolic variables (Table 3 ). The multiple regression analysis showed that all metabolic variables are significant explanatory variables for Rw4 and Rw8 (Table 4). According to the eigenvectors, the shape variable Rw4 was associated with an elongation of the end portion of the abdomen, due to positive movement on the y-axis of the landmarks 6 and 7 and to negative movement of landmark 8 on the same coordinate (Table 5). On the other hand, the shape variable Rw8 was associated to a shortening of the head, indicated by a negative movement on the $y$-axis of the landmark 1 tied to positive movements of the landmarks 2 and 3 on the same axis and to negative movements of the landmarks 4 and 5 producing a shortening of the abdomen (Table 5).

\section{DISCUSSION}

Animals need energy, which is generally scarce and in forms that are different than what is required by the organism. Since most energy-transforming processes are aerobic processes (Chown and Nicolson, 2004), animals need to transport oxygen and carbon dioxide through a series of limiting steps between the surrounding air and cells and tissues. In arthropods, respiratory systems provide an efficient mechanism of gas exchange, which depends on oxygen diffusion capacity through the anastomising tubules of tracheae (Snodgrass, 1993). This system works well at relatively small tracheal volumes (Chown and Nicolson, 2004; Willmer et al., 2005). Indeed, one of the most prominent hypotheses explaining the large body sizes of insects in the Paleozoic era is related to the fact that atmospheric oxygen concentration was 35\% at that time, supporting the idea that present insect size is limited by the present oxygen concentration (Kirkton, 2007). In fact, many researchers are interested in determining how and when size limitations affect insect respiration and metabolism in present-day insects (Hack, 1997; Davis et al., 1999; Chown and Nicolson, 2004; Hartung et al., 2004; Merrick and Smith, 2004; Greenlee and Harrison, 2005; Harrison et al., 2005; Nespolo et al., 2005; Kirkton, 2007; Nespolo et al., 2008). Given the constraints related to body size in animals with tracheae, it is reasonable to postulate that at larger body sizes, compensatory mechanisms, such as body deformations (Hartung et al., 2004), exist to improve the efficiency of respiration (Kirkton, 2007). Actually, in most insects, respiratory movements largely involve the thorax and the first segment of the abdomen (Snodgrass, 1993). But in orthopterans, the role of the abdomen appears to be more important because gas circulation occurs by changes in abdominal pressures of the haemolymph, due to abdominal contractions and expansions (i.e., in the dorso-ventral and longitudinal plane). Thus,

TABLE 3

Pearson's correlation coefficients between shape variables (relative warps 1 to 12 ) and metabolic variables. For abbreviations of metabolic variables see materials and methods. *Significant correlations at P-level $<0.05$

\begin{tabular}{lllllllllllll}
\hline & Rw1 & Rw2 & Rw3 & Rw4 & Rw5 & Rw6 & Rw7 & Rw8 & Rw9 & Rw10 & Rw11 & Rw12 \\
\hline AVG & 0.04 & 0.01 & 0.10 & $0.30^{*}$ & -0.12 & 0.15 & 0.01 & $-0.27^{*}$ & 0.12 & 0.01 & -0.21 & 0.05 \\
MIN & $-0.24^{*}$ & 0.00 & 0.06 & $0.36^{*}$ & -0.21 & 0.16 & 0.02 & $-0.36^{*}$ & -0.01 & -0.01 & 0.04 & -0.02 \\
MAX & 0.02 & -0.08 & -0.01 & $0.38^{*}$ & -0.21 & 0.01 & 0.03 & $-0.25^{*}$ & $0.33^{*}$ & -0.08 & -0.12 & 0.01 \\
REST & -0.08 & 0.03 & -0.02 & $0.43^{*}$ & -0.14 & 0.11 & 0.19 & -0.21 & $0.24^{*}$ & -0.03 & -0.08 & 0.03 \\
ACT & -0.17 & -0.03 & 0.02 & $0.27^{*}$ & -0.05 & -0.01 & -0.02 & $-0.24^{*}$ & 0.21 & $-0.25^{*}$ & -0.12 & 0.04 \\
\hline
\end{tabular}


TABLE 4

Forward stepwise multiple regression analyses between metabolic (dependent) and shape (explanatory) variables. Only the shape variables that were significantly correlated to metabolic variables were included in the analyses (see Table 3 )

\begin{tabular}{lcccccc}
\hline & Partial r & Slope & t-test & d.f. & P-level & Goodness of fit \\
\hline Average & & & & & & $\mathrm{F}_{(2,86)}=7.278 ; \mathrm{P}=0.001$ \\
Rw4 & 0.285 & 12.214 & 2.86 & 86 & 0.005 & \\
Rw8 & -0.254 & -39.585 & -2.546 & 86 & 0.013 & \\
Minimum & & & & & & $\mathrm{F}_{(3,85)}=9.617 ; \mathrm{P}<0.001$ \\
Rw4 & 0.325 & 1.685 & 3.465 & 85 & 0.001 & \\
Rw8 & -0.306 & -5.78 & -3.264 & 85 & 0.002 & \\
Rw1 & -0.23 & -0.303 & -2.453 & 85 & 0.016 & \\
Maximum & & & & & & $\mathrm{F}_{(3,85)}=9.740 ; \mathrm{P}<0.001$ \\
Rw4 & 0.338 & 14.049 & 3.615 & 85 & 0.001 & \\
Rw9 & 0.297 & 47.471 & 3.173 & 85 & 0.002 & \\
Rw8 & -0.22 & -33.236 & -2.349 & 85 & 0.021 & \\
Resting & & & & & & $\mathrm{F}_{(1,71)}=15.723 ; \mathrm{P}<0.001$ \\
Rw4 & 0.426 & 3.565 & 3.965 & 71 & $<0.001$ & \\
Activity & & & & & & $\mathrm{F}_{(1,87)}=4.837 ; \mathrm{P}=0.031$ \\
Rw4 & 0.229 & 7.29 & 2.199 & 87 & 0.031 & \\
\hline
\end{tabular}

TABLE 5

Eigenvectors (see text for details) of the $x$ - and $y$-coordinates landmarks on all relative warps.

These are adimensional indexes that indicate the importance of each coordinate pair to each relative warp

\begin{tabular}{ccccccccccccc}
\hline & Rw1 & Rw2 & Rw3 & Rw4 & Rw5 & Rw6 & Rw7 & Rw8 & Rw9 & Rw10 & Rw11 & Rw12 \\
\hline x1 & -1.1 & 1.2 & -9.9 & -9.3 & 2.2 & 12.0 & 16.7 & 16.0 & 72.9 & -71.9 & 221.4 & -1.1 \\
y1 & 1.8 & -9.4 & -0.2 & 46.8 & -7.5 & 5.9 & 1.5 & -226.6 & 77.1 & 16.7 & -1.8 & 19.9 \\
x2 & -4.3 & 1.0 & -2.8 & 11.9 & -6.7 & -11.3 & -42.6 & -34.6 & -69.7 & -162.0 & -318.0 & -39.9 \\
y2 & 10.7 & 8.6 & -15.3 & -31.6 & 17.4 & -14.5 & -18.0 & 261.1 & -102.4 & -59.2 & -3.2 & 229.7 \\
x3 & 2.2 & -3.4 & -5.1 & 5.5 & 8.3 & -9.4 & -30.5 & -2.6 & -92.5 & 320.2 & -164.6 & 38.7 \\
y3 & 10.7 & 6.7 & 8.8 & -33.0 & 9.1 & -2.3 & 15.1 & 241.8 & -69.4 & 21.5 & 8.9 & -274.6 \\
x4 & -4.8 & 18.8 & 14.5 & 17.3 & -42.5 & 32.9 & 58.2 & 42.5 & 47.4 & 248.6 & 248.3 & 53.1 \\
y4 & -19.4 & -10.4 & -4.8 & -13.7 & -40.7 & 8.4 & 43.2 & -198.0 & 32.0 & 58.1 & 17.0 & -298.9 \\
x5 & 9.0 & -16.0 & 12.5 & -24.3 & 26.6 & -34.4 & 57.8 & -8.5 & 81.2 & -351.2 & 61.6 & -45.8 \\
y5 & -14.9 & -13.5 & 16.3 & -9.7 & -5.3 & 17.7 & -42.3 & -144.9 & 87.7 & -31.2 & -24.2 & 330.3 \\
x6 & 8.9 & -19.0 & -3.2 & -21.5 & 50.8 & -12.5 & -67.0 & -24.2 & -44.2 & -32.1 & -56.9 & -14.0 \\
y6 & 7.1 & 26.9 & 14.7 & 53.0 & 46.8 & -11.4 & -21.0 & 80.3 & 11.0 & -9.4 & -7.7 & 43.2 \\
x7 & -7.8 & 13.6 & 1.3 & 18.2 & -19.1 & 41.9 & -75.2 & -8.3 & -51.6 & 61.0 & -25.8 & 2.4 \\
y7 & 2.4 & 31.2 & -22.7 & 50.5 & 24.0 & -29.1 & 22.4 & 47.6 & -61.1 & -2.3 & 16.2 & -56.8 \\
x8 & -2.1 & 3.6 & -7.3 & 2.2 & -19.6 & -19.2 & 82.5 & 19.6 & 56.5 & -12.5 & 34.1 & 6.7 \\
y8 & 1.7 & -40.1 & 3.1 & -62.3 & -43.7 & 25.5 & -1.0 & -61.3 & 25.1 & 5.8 & -5.0 & 7.2 \\
\hline
\end{tabular}


the significant correlation between energy metabolism and abdomen elongation found in this study has a functional basis: ventilation, which occurs across the longitudinal axis, would be more efficient by abdomen elongations. In other words, ontogenic shape variations would be compensatory mechanisms that improve respiratory efficiency at large sizes. For instance, Lease et al. (2006) described that tracheal volume decreases during growth within an instar, while it increases between instars, most likely due to displacement by growing tissues. These tissues comprise gonads, which certainly would compete with space with the respiratory tissue. It would be interesting, hence, to establish to what extent these two functions (i.e., respiration and reproduction) could be competing in later instars and adults.

During insect ontogeny, tracheal system capacities closely match metabolic rates (Joos et al., 1997; Suarez, 1998; Harrison et al., 2005; Heymann and Lehmann, 2006). Abdomen deformations would assist the restrictions imposed by size, but also must be in concordance with the development of other important structures such as gonads (see Crnokrak and Roff, 2002; Nespolo et al., 2008). Also, larger tracheae systems impose additional problems such as evaporative water loss (Quinlan and Hadley, 1993; Lehmann et al., 2000). Given that our results were obtained in non-reproductive individuals, we cannot comment about the potential conflicts that gonads and respiratory structures could exhibit. However, it would be interesting to explore how large adult insects respond to the potentially conflicting demands of reproduction, respiration and water economy.

There are several possible compensatory effects that we did not address, affecting body shape such as tracheolar differences between the different instars (Hartung et al., 2004), neural differences in responsiveness to atmospheric $\mathrm{O}_{2}$, energy demand or environmental variables that could affect body size and shape (e.g., temperature, humidity and food availability). These factors certainly open several avenues for further research. The present study, however, indicates that geometric morphometrics could be a powerful tool in helping to solve these problems, being a worthwhile and feasible area of investigation.

\section{ACKNOWLEDGEMENTS}

We thank Roberto Cipriani for valuable comments to the manuscript. R.D. Sepúlveda and L.E. Castañeda were supported by CONICYT-Chile Doctoral Fellowships. This work was partially funded by Anillos ACT-38 project.

\section{REFERENCES}

ADAMS DC (1999) Methods for shape analysis of landmark data from articulated structures. Evol Ecol Res 1: 959-970.

ADAMS DC, ROHLF FJ, SLICE DE (2004) Geometric morphometrics: ten years of progress following the 'revolution'. Ital J Zool 71: 5-16.

BOOKSTEIN FL (1991) Morphometric tools for landmark data. New York: Cambridge University Press.

BRAMBLE DM, JENKINS FA (1993) Mammalian locomotor-respiratory design, implications for diaphragmatic and pulmonary design. Science 262: $235-240$.

CRNOKRAK P, ROFF DA (2002) Trade-offs to flight capability in Gryllus firmus: the influence of whole-organism respiration rate on fitness. J Evol Biol 15: 388-398.

CHOWN SL, NICOLSON SW (2004) Insect physiological ecology. Oxford: Oxford.
DAVIS ALV, CHOWN SL, SCHOLTZ CH (1999) Discontinuous gas exchange cycles in Scarabelus dung beetles (Coleoptera: Scarabaeidae): massscaling and temperature dependence. Physiol Biochem Zool 72: 555-565.

DIXON AFG, KINDLMANN P (1999) Cost of flight apparatus and optimum body size of aphid migrants. Ecology 80: 1678-1690.

FEDER ME, BURGGREN WW (1985) Cutaneous gas exchange in vertebrates: design, patterns, control and implications. Biol Rev Cambridge Philosop Soc 60: 1-45.

GOULD SJ (1966) Allometry and size in ontogeny and phylogeny. Biol Rev 41: 587-640.

GREENLEE KJ, HARRISON JF (2005) Respiratory changes throughout ontogeny in the tobacco hornworm caterpillar, Manduca sexta. J Exp Biol 208: 1385-1392.

HACK MA (1997) The effects of mass and age on standard metabolic rate in house crickets. Physiol Entomol 22: 325-331.

HARRISON JF, LAFRENIERE JJ, GREENLEE KJ (2005) Ontogeny of tracheal dimensions and gas exchange capacities in the grasshopper, Schistocerca americana. Comp Biochem Phys A 141: 372-380.

HARTUNG DK, KIRKTON SD, HARRISON JF (2004) Ontogeny of tracheal system structure: A light and electron-microscopy study of the metathoracic femur of the American locust, Schistocerca americana. J Morphol 262: 800-812.

HEYMANN N, LEHMANN FO (2006) The significance of spiracle conductance and spatial arrangement for flight muscle function and aerodynamic performance in flying Drosophila. J Exp Biol 209 1662-1677.

JOOS B, LIGHTON JRB, HARRISON JF, SUAREZ RK, ROBERTS SP (1997) Effects of ambient oxygen tension on flight performance, metabolism, and water loss of the honeybee. Physiol Zool 70: 167-174.

KIRKTON SD (2007) Effects of insect body size on tracheal structure and function. In Hypoxia and the Circulation, Vol. 618, pp. 221-228. Lehman FO (2001) Matching spiracle opening to metabolic need during flight in Drosophila. Science 294: 1926-1929.

LEASE HM, WOLF BO, HARRISON JF (2006) Intraspecific variation in tracheal volume in the American locust, Schistocerca americana, measured by a new inert gas method. J Exp Biol 209: 3476-3483.

LEHMANN FO, DICKINSON MH, STAUNTON J (2000) The scaling of carbon dioxide release and respiratory water loss in flying fruit flies (Drosophila spp.). J Exp Biol 203: 1613-1624.

LIGHTON JRB, TURNER R (2004) Thermolimit respirometry: an objective assessment of critical thermal maxima in two desert harvester ants, Pogonomyrmex rugosus and P. californicus. J Exp Biol 207: 1903-1913.

MERRICK MJ, SMITH RJ (2004) Temperature regulation in bury in beetles (Nicrophorus spp.: Coleoptera: Silphidae): effects of body size, morphology and environmental temperature. J Exp Biol 207: 723-733.

NESPOLO RF, CASTAÑEDA LE, ROFF DA (2005) Dissecting the variancecovariance structure in insect physiology: the multivariate association between metabolism and morphology in the nymphs of the sand cricket (Gryllus firmus). J Insect Physiol 51: 913-921.

NESPOLO RF, CASTAÑEDA LE, ROFF DA, FAIRBAIRN DJ (2008) Energetic trade-off between maintenance costs and flight capacity in the sand cricket (Gryllus firmus). Funct Ecol 22: 624-631.

QUINLAN MC, HADLEY NF (1993) Gas exchange, ventilatory patterns, and water loss in two lubber grasshopper: quantifying cuticular and respiratory patterns. Physiol Zool 66: 628-642.

ROGOWITZ GL, CHAPPELL MA (2000) Energy metabolism of eucalyptusboring beetles at rest and during locomotion: gender makes a difference. J Exp Biol 203: 1131-1139.

ROHLF FJ (2005a) tpsDig2. New York: SUNY.

ROHLF FJ (2005b) tpsRelw. New York: SUNY.

ROHLF FJ (1999) Shape statistics: Procrustes superimpositions and tangent spaces. J Classif 16: 197-223.

ROHLF FJ, SLICE D (1990) Extensions of the Procrustres method for the optimal superimposition of landmarks. Syst Zool 39: 40-59.

SLICE DE (2001) Landmark coordinates aligned by Procrustes analysis do not lie in Kendall's shape space. Syst Biol 50: 141-149.

SNODGRASS RE (1993) Principles of insect morphology: Comstock, Cornell University Press. First published, 1935 by McGraw Hill Book Company, Inc.

SUAREZ RK (1998) Energy metabolism during insect flight: biochemical design and physiological performance. Physiol Biochem Zool 73: 765771.

WILLMER P, STONE G, JOHNSTON IA (2005) Environmental physiology of animals. Malden: Blackwell.

ZAR JH (1999) Biostatistical Analysis. Fourth edition, Prentice Hall, New Jersey. 